W.P. 07-06

\title{
Experimental Economics: Contributions, Recent Developments, and New Challenges
}

\author{
Marie-Claire Villeval
}

Mars 2007

GATE Groupe d'Analyse et de Théorie Économique UMR 5824 du CNRS

93 chemin des Mouilles - 69130 Écully - France

B.P. $167-69131$ Écully Cedex

Tél. +33 (0)4 72866060 - Fax +33 (0)4 72866090

Messagerie électronique gate@gate.cnrs.fr

Serveur Web : www.gate.cnrs.fr 
Forthcoming in French Politics

\title{
Experimental Economics: Contributions, Recent Developments, and New Challenges
}

\author{
Marie-Claire Villeval
}

\begin{abstract}
Although economics has long been considered as a non-experimental science, the development of experimental economics and behavioral economics is amazingly rapid and affects most fields of research. This paper first attempts at defining the main contributions of experiments to economics. It also identifies four main trends in the development of experimental research in economics. The third contribution of this paper is to identify the major theoretical and methodological challenges faced by behavioral and experimental economics.
\end{abstract}

JEL-code: A120, C900, D010, D020

Key words: experimental economics, neuroeconomics, quantitative methods, field experiments

GATE; CNRS; University of Lyon; Ecole Normale Supérieure LSH

GATE, 93 chemin des Mouilles, 69130 Ecully (France)

Tel: +33 4728660 79. Fax: +33 4728660 90. Email: villeval@gate.cnrs.fr 
Economics was traditionally considered as a non-experimental science that must rely on direct observation of the world because of a supposed lack of control for important aspects of economic decision-making (Samuelson and Nordhaus, 1985 cited by Friedman and Sunde, 1994). This view has been challenged by the development of behavioral economics (Camerer, 2003) that largely relies on experimental evidence. The laboratory offers a controlled environment that allows researchers to measure the impact of exogenous institutional changes while guaranteeing ceteris paribus conditions. The attribution of the Prize in Economic Sciences 2002 to V. Smith for his research works on market mechanisms and to D. Kahneman for having integrated insights from psychology into economics has consecrated this change in perspective.

Experiments are now carried out in most economic fields, including macroeconomics, and the number of publications incorporating experimental work is blooming. This enthusiasm for experiments in economics is partly justified by a willingness to test the predictions delivered by game-theoretical models and by decision theory. The repeated observation of anomalies in the lab has given rise to the conviction that behavior is not only and not always driven by money maximization but that utility is also affected by emotions and affect and that economic reasoning depends on cognitive conditions. This has provoked a need for further explorations. Another reason for the success of experimental economics can be found both in the search for the appropriate counterfactual and in the difficulties faced by researchers to get access to survey data.

This paper does not aim at presenting a state-of-the-art of this method in economics but attempts at identifying how this method is contributing to the renewal of research and knowledge in economics. In a first section, it briefly recalls its major findings and emphasizes the diversity and the richness of the protocols tested. In a second section, it tries to identify new tendencies in the 
use of the experimental method in economics. In a last section, it presents a reflection about the theoretical and methodological challenges faced by this method.

\section{A major contribution of experiments to economics}

V. Smith has emphasized three functions of experiments in economics: testing theories, generating data and contributing to institutional design. Regarding the first two functions, major contributions have been made in the analysis of markets under perfect or imperfect information, in the understanding of individual decision-making (loss aversion, endowment effect, ambiguity aversion, law of small numbers, preference reversal, ...) and in the study of social interactions (altruistic punishment, trust, fairness, inequality aversion, ...). Some myths have then been challenged by the experimental research (Bazerman and Malhotra, 2006): in particular, preferences are not always stable and consistent; the agents do not have perfect knowledge of their preferences; they do not always use all the available information to make decisions. This is especially striking in the analysis of incentives. Many studies have shown the impact of incentives on the individual behavior in terms of effort decisions, as predicted by the agency theory; we have however accumulated many proofs of evidence that in some circumstances, incentives may have a negative impact on the effort level chosen by the agents (incentives may crowd-out intrinsic motivation; they may be ineffective in the presence of income targeting; etc.). Indeed, testing economic theory, experimental research has identified several limitations to strategic thinking: these limitations are related to the existence of social motivations, of psychological and judgmental biases, of limited cognitive abilities and emotions. On the other side, other experiments have simultaneously confirmed the strong influence of market forces and 
competition on human decisions. The coexistence of these results influences the agenda of further research.

The contribution of lab experiments to institutional design is also noticeable. Experiments are able to address several public policy questions. They can be used by companies that plan to reform their organization, their strategy on the market, or their human resources policy. Experiments provide useful tests of alternative market designs (for example, the design of auctions of broadcast bandwidths or the organization of pollution permit markets). They offer a cheap alternative to small-scale policy implementation. Progress has also been made in the analysis of various funding modes of collective goods or political campaigns (for example, Houser and Stratmann, 2006). Another contribution is related to the fact that experiments can help in analyzing the agents' preferences when markets do not exist (for example, approaching the relationship between valuation and choices, or analyzing tax compliance under various auditing regimes). The extent to which policy makers incorporate experimental evidence into policy design is however less clear.

One should now emphasize a fourth contribution of experimental economics: it offers academics an innovative teaching tool. Classroom experiments have expanded dramatically in the 90s, thanks to prominent contributors such as C. Holt (see his Veconlab Software: http://veconlab.econ.virginia.edu/admin.htm, and his recent book on Markets, Games, and Strategic Behavior, 2006). Leading journals, such as the Journal of Economic Literature, include a section devoted to the practical organization of classroom experiments. This evolution has enabled a more active learning process in economics, based on the self-discovery by students of fundamental economic notions, reconciling abstract mathematical modeling with empirical 
observations (see Holt, 2003). It certainly contributes to counteract the distaste of students for majoring in economics. Several examples of classroom experiments on voting, political institutions, and agenda effects are provided in these journals and website.

\section{New tendencies}

The development of its interactions with other social sciences, in particular psychology, characterizes the recent evolution of experimental economics. In particular, behavioral game theory can be seen as a willingness to blend « experimental evidence and psychology in a mathematical theory of normal strategic behavior » (Camerer, 2003). More and more experimental economists pay attention to the work of social psychologists, and vice-versa (de Cremer, Zeelenberg, and Murnighan, 2006). This leads to the import of insights from behavioral decision research into economic analysis, for a better understanding of motivation, social interactions and individual choices.

To a smaller extent, this dialogue has also developed with anthropology, in particular to investigate whether economic behavior is transversal to cultures and to various stages of development (see the replication on the field of standard economic games in small-scale societies by Henrich et al., 2004). Cooperation between experimental economists and political scientists should develop quite easily, regarding the analysis of voting behavior (see the numerous papers in the economics of voting by T.R. Palfrey, such as for example Levine and Palfrey, 2006, or Aragones and Palfrey, 2005) or decision-making in committees (in the tradition of Fiorina and Plott, 1978). 
Despite these fruitful interactions, it should however be noted that important methodological differences remain, especially regarding the role of monetary incentives, the status of deception and the use of context (see Croson, 2006; Hertwig and Ortmann, 2002).

A second major evolution of experimental economics is related to the analysis of rationality and its limitations, thanks particularly to the development of neuroeconomics (brain imaging, EEG, PET and fMRI) and physionomics (skin conductance measures, heart rate, measures of hormones, study of pupil dilation). Opening the "black box of brain activity" (Camerer, Loewenstein, and Prelec, 2005) aims at establishing the role of controlled processes (deliberation and learning) vs. automatic processes that are below consciousness in economic decision-making. It also aims at analyzing the brain during decision-making to inform about the relationships between the ('hot') affective and ('cold') cognitive processes involved in performing an economic task. These works lead to questions basic standard assumptions in economics regarding choice and social interactions and contribute to a better understanding of the importance of limited foresight and emotions in economic decisions.

A third direction of evolution is the reinforcement of the realism of economic experiments and the diversification of protocols. Indeed, the external validity of experiments is still an issue. The sterility of the lab environment has many advantages, regarding the conditions of control and the measure of exogenous changes, but it raises some concerns regarding the possibility to extrapolate evidence collected in the lab to real settings. One can also criticize the lack of variability of socio-demographic characteristics of the standard student-subject pools or their lack of expertise. A partial answer can be found in the current development of field experiments, probably initiated by Levine and Plott (1978) in their study of the impact of agenda manipulation 
on voters' decisions. Whatever artefactual, framed or natural, field experiments constitute an important complement of lab experiments to allow general inferences (Harrison and List, 2004). The reinforcement of realism can also be achieved by combining experimental tests with survey data (see for example the call for contribution to the ANES Panel Study on voting behavior). We also observe a diversification of subject-pools, an increase in the sample size (facilitated by notably the development of internet-based experiments), or the use of representative samples (Harrison, Lau, and Williams, 2002). This is accompanied by the design of less abstract tasks, the elicitation of valuation instead of induced valuation through virtual goods, the import of parameters values from the field. This evolution is however not without raising other potential concerns (loss of control for the cost of effort, risk of uncontrolled selection or recruitment biases, ...).

\section{Major theoretical and methodological challenges}

Experimental research has revealed many anomalies in behavior that refute the predictions delivered by economic theory. However, as pointed out by Camerer (2006), «the goal of behavioral economics is not just to create a list of anomalies. The anomalies are used to inspire and constrain formal alternatives to rational-choice theories ». These anomalies do not condemn economic theory per se but they certainly call for a transformation of the theory and a search for consistency. This raises of course a more fundamental question regarding the nature of the revision process. One possibility is to work on updating standard theory. For example, experiments have widely documented the importance of fairness in economic transactions. A possibility would be to incorporate additional constraints (for example an equity constraint) in the design of contracts, but the general framework of the agency theory would be preserved. An 
alternative is to develop a theory that would constitute an alternative to the rational choice theory and that would explain conflicting behaviors in various contexts. This option is explored by the so-called dual theories, opposing controlled, “cold”, or deliberative processes to automatic, "hot”, or affective processes (see Camerer, Prelec, and Loewenstein, 2005; Loewenstein and O’Donoghue, 2006), or by the cognitive hierarchy models in which each player assumes that his strategy is the most sophisticated (Camerer, Ho, and Chong, 2004).

Among the theoretical challenges that need to be addressed by further research, a preferential treatment should be given to the following questions. Should one incorporate social preferences, and non-monetary sources of motivation in the utility function and where should one put the limit in the manipulation of this function? When do market forces dominate and when do social preferences dominate? When do selfish people contaminate the socially-oriented subjects? Some suggest that in games where strategies are complements, a small number of socially-oriented subjects may change the behavior of selfish people (Camerer and Fehr, 2006). Answering this fundamental question requires to understand why «non-standard » behavior (i.e. non predicted by rational-choice theory) survives, declines or develop. This calls for a development of research on the market implications of emotions and on how the market "disciplines" emotions. More largely, we need to study the relationships between emotions or affective decision-making and efficiency. Another important theoretical challenge is to determine what should be the status of agents' heterogeneity in preferences or abilities. Most experiments reveal a diversity of preferences and orientations that is not usually predicted by the theory, and that may have an impact on optimal matching in groups. Either one considers this diversity as pure empirical noise or one tries to give a status to heterogeneity in economic theory. Allowing for heterogeneity also 
means that sorting and self-selection matter. We should therefore also investigate the interactions between self-selection, emotions and rationality in decision-making.

From a methodological point of view, many questions regarding the collection of data remain unanswered. Do we test mean behavior or do we search for limit cases? A challenge is that a lab experiment must reproduce a theoretical model but it must be at the same time able to mimic the field context reliably. These two orientations may not be necessarily compatible: one can consider that a pragmatic attitude must prevail (the design of an experiment must depend on the research issue), but one can also consider that it is an epistemological issue (experiments aim at reproducing models and parallelism is a secondary issue). The discussion is still open. In the same vein, how can one interpret the differences revealed by the comparison of field and lab experiments? It is important to be able to determine whether they are related to differences in values, in optimization variables, or in strategies. Another point is related to the grasp of time in lab experiments, especially since we are aware of time-inconsistency in human decisions (O’Donoghue and Rabin, 2007). But how can one reproduce medium-term or long-term in the lab (Gneezy and List, 2006), and how can one reproduce infinite-horizon games? The next point is related to the methodological implications of collected evidence on the impact of smartness and mood on behavior. It probably suggests that we should increase the control for subjects' smartness, attention, and mentalizing abilities. Similarly, a control for mood may be justified in some experiments since it may influence affective reasoning (Kirchsteiger, Rigotti, Rustichini, 2006). In the same perspective, it matters to collect more data on the individual background of the experimental subjects to control for possible sample biases (for example personal wealth and social origin are likely to influence behavior in bargaining games; the number of siblings may affect decisions in public good or coordination games; etc.). Another concern may however 
arise: to which extent can we collect additional and peripheral information about our subjects' abilities and background without polluting behavior in the game? A fourth point is related to the measure of emotions. When is it sufficient to use self-reported measures and when should one use physiological measures?

The statistical analysis of experimental data requires also further improvements. Although some scholars still believe that non-parametric statistics constitute the best method to analyze experimental data, more and more numerous are those who consider that the analysis of experimental data benefits from the application of suitable econometric tools in addition to nonparametric statistics, in particular the econometrics of panel data. The development of « experimetrics» (Bardsley and Moffatt, 2006) allowed by a deeper cooperation between experimentalists and econometricians would enable scholars to improve the techniques adapted to the specific nature of the experimental data (in particular, the existence of small samples with a few independent observations but repeated observations). In particular, there is a need for the development of techniques enabling a treatment of the potential selection biases in repeated sequential games, or appropriate to the analysis of the diversity of strategies (for example, the socalled finite mixture models).

\section{Conclusion}

In sum, experimental economics constitutes a powerful method to develop knowledge in economics. It helps in identifying empirical regularities and so doing it inspires new research questions. It contributes to the renewal of economic theory by incorporating emotions, affect and cognition in the analysis of behavior. This evolution has been favored by a more open-minded 
attitude towards other disciplines, in particular psychology and neurosciences. This buoyant field of research fosters excitement for this method and the competition between experimental economists contributes to the rapid development of knowledge in the field of behavioral economics. On the downside of this evolution is the observation that experiments revealing anomalies raise more curiosity and attention than those that do not refute theory, but do not usually attempt to revise the theory. Another drawback is that it is more exciting for scholars to test new protocols than to replicate previous ones, since it pays less to replicate a protocol than to design a new experiment. Therefore, if one considers that replication is crucial for certifying the robustness of scientific results, it would be suitable to provide more incentives for the replication of experiments, in particular in terms of publications in high-rank economic journals. 


\section{REFERENCES}

Aragones, E., Palfrey, T.R. (2005). « Spatial Competition Between Two Candidates of Different Quality: The Effects of Candidate Ideology and Private Information. » In Social Choice and Strategic Behavior: Essays in Honor of Jeffrey Scot Banks. D. Austen-Smith and J. Duggan (Eds.) Berlin: Springer.

Bardsley, N., Moffatt, P.G. (2006). The Experimetrics of Public Goods: Inferring Motivations from Contributions. Cedex Discussion Paper, 2005-09, Nottingham.

Bazerman, M.H., Malhotra, D. (2006). Economics Wins, Psychology Loses, and Society Pays. In Social Psychology and Economics, D. de Cremer, J.K. Murnighan and M. Zeelenberg (Eds.), Mahwah: Lawrence Earlbaum Associates. 263-280.

Camerer, C.F. (2003). Behavioral Game Theory: Experiments in Strategic Interaction. Princeton: Princeton University Press.

Camerer, C.F. (2006). Behavioral Economics. World Congress of the Econometric Society, London.

Camerer, C.F., and Fehr, E. (2006). When Does 'Economic Man' Dominate Social Behavior? Science, 311, January.

Camerer, C.F., Loewenstein, G., Prelec, D. (2005). Neuroeconomics: How Neuroscience can Inform Economics. Journal of Economic Literature, XLIII (1), 9-64.

Camerer, C.F., Ho, T.H., and Chong, J.K. (2004). A Cognitive Hierarchy Theory of One-shot Games. Quarterly Journal of Economics, 119(3), 861-898.

de Cremer, D., Murnighan, J.K., and Zeelenberg, M. (Eds.) (2006). Social Psychology and Economics. Mahwah: Lawrence Earlbaum Associates.

Croson, R. (2006). Contrasting Methods and Comparative Findings in Psychology and Economics. In Social Psychology and Economics, D. de Cremer, J.K. Murnighan and M. Zeelenberg (Eds.), Mahwah: Lawrence Earlbaum Associates.

Fiorina, M., Plott, C. (1978). Committee Decisions under Majority Rule: An Experimental Study. American Political Science Review,72, 575-598.

Friedman, D., Sunder, S. (1994). Experimental Methods: A Primer for Economists. Cambridge : Cambridge University Press.

Gneezy, U., List, J.A. (2006). Putting Behavioral Economics to Work: Testing for Gift Exchange in Labor Markets using Field Experiments. NBER WP12063.

Harrison, G.W., Lau, M.L., and Williams, M.B. (2002). Estimating Individual Discount Rates in Denmark: A Field Experiment. American Economic Review, 92(5), 1606-1617.

Harrison, G.W., List, J.A. (2004). Field Experiments. Journal of Economic Literature, 42(4), 1013-1059.

Henrich, J., Boyd, R., Bowles, S., Camerer, C., Fehr, E., and Gintis, H. (Eds.) (2004). «Foundations of Human Sociality: Economic Experiments and Ethnographic Evidence from Fifteen Small-Scale Societies. » Oxford : Oxford University Press. 
Hertwig, R., Ortmann, A. (2002). Economists' and psychologists' experimental practices: How they differ, why they differ and how they could converge. In The psychology of economic decisions, I. Brocas and J. D. Carillo (Eds.), New York: Oxford University Press, 253-272.

Holt, C.A. (2003). Economic Science: An Experimental Approach for Teaching and Research. Presidential Address to the Southern Economic Association, Southern Economic Journal, 69(4), 755-771.

Holt, C.A. (2006). Markets, Games, and Strategic Behavior. Addison-Wesley.

Houser, D., Stratmann, T. (2006). Selling Favors in the Lab: Experiments on Campaign Finance Reform. CESifo Working Paper Series No. 1727.

Kirchsteiger, G., Rigotti, A., Rustichini, A. (2006). Your Morals are Your Moods. Journal of Economic Behavior and Organization. 59, 155-172.

Levine, D., Palfrey, T.R. (2006). The Paradox of Voter Participation: A Laboratory Study. American Political Science Review, forthcoming .

Levine, M., Plott, C. (1978). A Model of Agenda Influence on Committee Decisions. American Economic Review, 68, 146-160.

Loewenstein, G., O’Donoghue, T. (2006). 'We Can Do This the Easy Way or the Hard Way': Negative Emotions, Self-regulation and the Law. University of Chicago Law Review, 73, 183206.

O’Donoghue, T., Rabin, M. (2007). Procrastination on Long-Term Projects, Journal of Economic Behavior and Organization, forthcoming.

Samuelson, L. (2005). Economic Theory and Experimental Economics. Journal of Economic Literature, XLIII (1), 65-107.

Samuelson, P.A., Nordhaus, W.D. (1985). “Economics.” $12^{\text {th }}$ edition. McGraw-Hill.

Smith, V. (1962). An Experimental Study of Competitive Market Behavior. Journal of Political Economy.

Smith, V. (2002). Method in Experiment: Rhetoric and Reality. Experimental Economics, 5(2), 91-110. 